\title{
RTKN2 is Associated with Unfavorable Prognosis and Promotes Progression in Non-Small-Cell Lung Cancer
}

\author{
Lupeng Ji ${ }^{1, *}$ \\ Yucun Huang ${ }^{2, *}$ \\ Yi Zhang ${ }^{3, *}$ \\ Anping Peng ${ }^{4}$ \\ Jilong $\operatorname{Qin}^{5}$ \\ Shaofan $\mathrm{Lu}^{2}$ \\ Yu Huang ${ }^{6}$
}

'Department of Medicine, The Fifth People's Hospital of Zhuhai, Zhuhai 5 I 9055, People's Republic of China; ${ }^{2}$ Department of Radiology, The Fifth People's Hospital of Zhuhai, Zhuhai 519055, People's Republic of China; ${ }^{3}$ Department of Respiratory Medicine, Zhuhai People's Hospital, Zhuhai 519000, People's Republic of China; ${ }^{4}$ South Campus Clinic, Sun Yat-sen Memorial Hospital, Sun Yat-sen University, Guangzhou 510120, People's Republic of China; ${ }^{5}$ Department of Pathology, The First Affiliated Hospital of Guangzhou Medical University, Guangzhou 510120, People's Republic of China; ${ }^{6}$ Division 6 of East Ward (Respiratory), Guangdong Provincial People's Hospital, Guangdong Academy of Medical Sciences, Guangdong Provincial Geriatrics Institute, Guangzhou 510080, People's Republic of China

*These authors contributed equally to this work
This article was published in the following Dove Press journal: OncoTargets and Therapy

Background: Non-small-cell lung cancer (NSCLC) is the leading cause of cancer-related mortality worldwide. However, the molecular mechanism of NSCLC remains unknown. Accumulating data show that Rhotekin 2 (RTKN2) functions as a novel crucial regulator of diverse biological processes; however, its pathological role in NSCLC remains unclear.

Methods: In this study, we investigated the function of RTKN2 in NSCLC. The expression of RTKN2 mRNA was analyzed in tumor tissues and paired adjacent tissues from patients by qRT-PCR. The role of RTKN2 in cell proliferation, apoptosis, migration, and invasion was investigated. The potential mechanisms were explored.

Results: We found that the level of RTKN2 mRNA was up-regulated in NSCLC tissues and cell lines. RTKN2 knockout inhibited the proliferation of human NSCLC cell lines A549 via inducing apoptosis by increasing the level of Bax and decreasing the level of Bcl-2. Furthermore, silencing of RTKN2 reduced the migration and invasion of A549 cells through up-regulated matrix metalloproteinase-9 (MMP9) and MMP2 expression.

Conclusion: These data suggest that RTKN2 may not only be a prognostic biomarker candidate but also provide a potential therapeutic target for NSCLC diagnosis and treatment. Keywords: RTKN2, proliferation, apoptosis, migration, invasion, non-small-cell lung cancer

\section{Background}

Lung cancer accounts for the main cause of cancer-related mortality around the world. ${ }^{1}$ Despite the success of targeted and/or immune-based therapies, the 5-year survival of patients is $55.6 \%$ but only $4.5 \%$ for metastatic disease, and early diagnosis and surgical resection provide the best opportunity for survival. ${ }^{2,3}$ Non-small cell lung cancer (NSCLC) accounts for $85 \%$ of cases. ${ }^{1}$ At present, NSCLC is defined histopathologically in the clinic into four broad categories: lung adenocarcinoma, lung squamous cell carcinoma, large cell carcinoma, and undifferentiated NSCLC. ${ }^{4}$ Lung adenocarcinoma is the most frequent histological subtype of NSCLC. The incidence of and mortality rates for NSCLC are rising globally on an annual basis. Despite progress in early diagnosis and targeted therapy, the clinical outcome of NSCLC patients remains poor, which is mainly due to patients usually being diagnosed at advanced disease stages. 5,6 Thus, further intensive research efforts into the molecular mechanisms of NSCLC could improve patient survival. ${ }^{7}$

The Rho GTPases are members of the RAS superfamily, regulating many cellular processes. ${ }^{8}$ Rhotekin (RTKN), a Rho effector, was initially isolated as a
Correspondence: Yu Huang

Division 6 of East Ward (Respiratory), Guangdong Provincial People's Hospital, Guangzhou 510080, People's Republic of China

Email yhuangmd@I63.com
OncoTargets and Therapy 2020:13 10729-10738

10729

DovePress if in $>$ 
scaffold protein interacting with the GTP-bound form of Rho. ${ }^{9}$ Previous studies have shown that RTKN2 plays a critical role in the pathological process of cell proliferation, apoptosis, and invasiveness in various malignant tumor cells. ${ }^{10-13}$ Down-regulation of RTKN2 in hepatocellular carcinoma cells notably induced cell apoptosis, while significantly repressing cell invasion. ${ }^{12}$ The inhibitory effect of RTKN2 silencing on the proliferation of colon cancer cells may be partially realized by inhibiting the $W n t / \beta$-catenin signaling pathway. ${ }^{10}$ In addition, RTKN2 is involved in the progression of human osteosarcoma. ${ }^{11}$ These findings suggest an involvement of RTKN2 in tumor progression. However, the biological functions of RTKN2 in human NSCLC remain unclear.

In the present study, we aimed to examine the relationship between RTKN2 and NSCLC progression. Our findings suggested that RTKN2 expression was higher in tumor tissues than in paratumor tissue, and its expression was also related to TNM stage and lymphocyte metastasis. Functional studies have shown that RTKN2 knockout reduces the proliferation, migration, and invasion, and enhanced the apoptosis of NSCLC cells, and the opposite result was observed with RTKN2 knockout. Thus, our results showed that RTKN2 inhibited NSCLC progression and served as a potential tumor target gene, which will provide a new strategy for the treatment of NSCLC.

\section{Methods}

\section{Patients}

Twenty pairs of frozen fresh human NSCLC tissues, as well as their corresponding adjacent non-tumor tissues (paratumor), were collected in the First Affiliate Hospital of Guangzhou Medical University (Guangzhou, China) from 2014 to 2016. The patients were classified by two independent pathologists. This study was approved by the Ethics Committee of the Fifth People's Hospital of Zhuhai, the First Affiliate Hospital of Guangzhou Medical University, and Guangdong Provincial People's Hospital. Written informed consent was provided by all patients.

\section{Cell Culture and Transfection}

A549 and SPC-A1 cell lines were purchased from the Cell Bank of the Chinese Academy of Sciences (Shanghai, China) and maintained in Dulbecco's modified Eagle's medium (DMEM) (Gibco, Rockville, MD, USA) with 10\% heatinactivated fetal bovine plasma at $37^{\circ} \mathrm{C}$ in $5 \% \mathrm{CO}_{2}$. BEAS2B (American Type Culture Collection, USA), human normal osteoblast cells, were cultured in DMEM according to the providing sources. For RTKN2 knockout specifically, siRNAs against RTKN2 were designed and constructed by Geneseed Co. Ltd (Guangzhou, China), and the sequence is given as follows: siRTKN2-1, 5'-GCU UUG GUA GUA CCC AUU ATT-3'; siRTKN2-2, 5'-GCU UUG GUA GUA CCC AUU ATT-3'; siRTKN2-3, 5'-CCU UCU GGC AGC AUU UCU UTT-3'. Transfection was performed by the Lipofectamine 3000 (Invitrogen, Camarillo, CA, USA), according to the manufacturer's protocols. The efficiency of RTKN2 knockout was confirmed by qRT-PCR and Western blot assay. After $48 \mathrm{~h}$ of transfection, cells were collected for further analysis.

\section{MTT Assay}

The MTT assay was performed using a 3-(4,5dimethylthiazol-2-yl)2,5-diphenyltetrazolium bromide (MTT) assay kit (Beyotime, China). In brief, the transfected cells were seeded into 96-well culture plates at an initial density of $5 \times 10^{3}$ cells per well. After incubating for the indicated time, cells were treated with $100 \mu \mathrm{L}$ MTT reagent for another $4 \mathrm{~h}$ at $37^{\circ} \mathrm{C}$. Then, the formazan crystals were dissolved in DMSO. After incubation at $37^{\circ} \mathrm{C}$, the absorbance at $490 \mathrm{~nm}$ was measured in a microplate reader (PerkinElmer, Waltham, MA, USA).

\section{Colony Formation Assay}

After transfection, cells were seeded in the six-well plates at the density of 600 cells, then incubated for 10-14 days until the clone spots were visible. The resulting colonies were fixed with paraformaldehyde for $10 \mathrm{~min}$, then stained with crystal violet (Beyotime) for $15 \mathrm{~min}$; and visible cell colonies were then counted. At least three independent experiments were performed.

\section{Apoptosis Assay}

Apoptosis was measured by the Annexin V FITC Apoptosis Detection kit (Beyotime) according to the protocol. In brief, cells were transfected as described above, then collected, washed with ice-cold PBS and resuspended in $400 \mu \mathrm{L}$ binding buffer, containing $5 \mu \mathrm{L}$ Annexin-V FITC and PI, following which the cells were incubated for $15 \mathrm{~min}$ at room temperature in the dark prior to flow cytometry (BD Biosciences, USA).

\section{Cell Migration Assay}

For migration assays, $1 \times 10^{5}$ cells in $200 \mu \mathrm{L}$ of DMEM were placed into the upper chamber, and $600 \mu \mathrm{L}$ DMEM 
containing $10 \% \mathrm{FBS}$ in the lower chambers. After $24 \mathrm{~h}$ culture, cells on the top side were scraped off gently, then fixed in paraformaldehyde and stained with crystal violet. Three random microscopic fields were counted per field for each group under a light microscope.

\section{Cell Invasion Assay}

A 24-well transwell plate was used to measure the invasive ability of cells. Chamber inserts were precoated with $50 \mu \mathrm{L}$ of a 1:8 mixture of BD Matrigel (BD Biosciences, USA) and DMEM. Then, $2 \times 10^{5}$ cells were seeded in the upper chamber. After $48 \mathrm{~h}$, cells on the top side were scraped off, then fixed in paraformaldehyde, and stained with crystal violet. Three random microscopic fields were counted per field for each group under a light microscope.

\section{RNA Preparation and qRT-PCR}

RNA preparation was performed as previously described. ${ }^{14}$ Total RNA was extracted from 20 pairs of tumor and corresponding adjacent non-tumor tissues using RNAiso Plus reagent (Takara, China), and reverse transcription was performed using Primescript RT Master Mix (Takara). For qRT-PCR analysis, cDNA was amplified using an SYBR Green Kit (Takara). Data were analyzed using the $2^{-\Delta \Delta C T}$ method. The sequences used for amplification were as follows: RTKN2 forward (F): 5'-ACAGTTCGCGTTGGAG ATGGAG-3', RTKN2 reverse (R), 5'-GTCGAGCATTGC ACACCATGAG-3'; Bax F, 5'-ATCCAAGACCAGGG TGGTT-3', Bax reverse, 5'-ATCTGGAAGAAGATGGG CTG-3'; Bcl-2 F, 5'-GGCCTCTGTTTGATTTCTCC-3', Bcl-2 R, 5'-AGTGAAGTCAACATGCCTGC-3'; MMP9 (matrix metalloproteinase-9) F, 5'-TGTACCGCTATGGTT ACACTCG-3', MMP9 R, 5'-GGCAGGGACAGTTGC TTCT-3'; MMP2 F, 5'-CCCACTGCGGTTTTCTCGAAT3', MMP2 R, 5'-CAAAGGGGTATCCATCGCCAT-3'; $\beta$ actin F, 5'-GAAATCGTGCGTGACATTAA-3', $\beta$-actin F: 5'-AAGGAAGGCTGGAAGAGTG-3'.

\section{Protein Extraction and Western Blot Assay}

In brief, the total proteins were lysed using RIPA lysis buffer (Beyotime). After centrifugation at 12,000 $\mathrm{g} / \mathrm{min}$ for $20 \mathrm{~min}$, the supernatant was collected and the concentration was measured by the BCA assay (Beyotime). Equal amounts of protein were separated using SDS-PAGE, then transferred to a PVDF membrane. After blocking with non-fat milk for $1 \mathrm{~h}$ at room temperature, the membranes were incubated overnight with primary antibodies against RTKN2 (1:1000; Abcam), Bax (1:1000; Abcam), Bcl-2 (1:1000; Abcam), and MMP9 $\left(1: 1000 ;\right.$ Abcam) at $4^{\circ} \mathrm{C}$. $\beta$-Actin $(1: 5000$; Cell Signaling Technology) was used as a loading control. The membranes were subsequently washed three times with TBST, then incubated with secondary antibody for $1 \mathrm{~h}$. The bands were visualized with ECL and quantified using ImageJ.

\section{Statistical Analysis}

All statistical analysis was performed by SPSS 17.0 software (SPSS, Chicago, IL, USA). We used GraphPad Prism 7.0 (La Jolla, CA, USA) for image editing. The significant differences between groups were analyzed using the Student's $t$-test or Fisher's exact test. Overall survival (OS) curves were determined by the Kaplan-Meier method and log-rank test. A $P$-value $<0.05$ was considered statistically significant.

\section{Results}

\section{RTKN2 Is Up-Regulated in NSCLC}

First, to identify RTKN2, we examined its mRNA expression in NSCLC tissues and cells by qRT-PCR. It was confirmed that RTKN2 mRNA expression was up-regulated in NSCLC tumor tissues compared to the corresponding adjacent non-tumor tissues (Figure 1A). The RTKN2 expression levels were higher in $65.00 \%$ of NSCLC tumor tissue samples (Figure 1B). Then, we aimed to determine whether a high level of RTKN2 was associated with clinicopathological characteristics in NSCLC patients. Based on the TNM stage, RTKN2 mRNA expression was significantly associated with NSCLC patient pathogenesis (Figure 1C). The RTKN2 expression level was also closely related to lymphatic metastasis (Figure 1D). RTKN2 mRNA expression showed no significant differences between groups of different sexes, ages, and smoking status. TNM stage and lymphatic metastasis were correlated with the RTKN2 mRNA expression level using Fisher's exact test $(P=0.022$, $P=0.017$, respectively), as shown in Table 1 . Next, further survival analysis showed that the high RTKN2 expression group $(n=13)$ had shorter OS time than the low RTKN2 expression group $(n=7)$ (Figure 1E). Then, we assessed RTKN2 expression in NSCLC cell lines. The RTKN2 level was increased in SPC-A1 and A549 NSCLC cell lines compared to the normal osteoblast cell line BEAS$2 \mathrm{~B}$ (Figure 1F-H). These data suggest that abnormal RTKN2 activation may be related to NSCLC progression. 

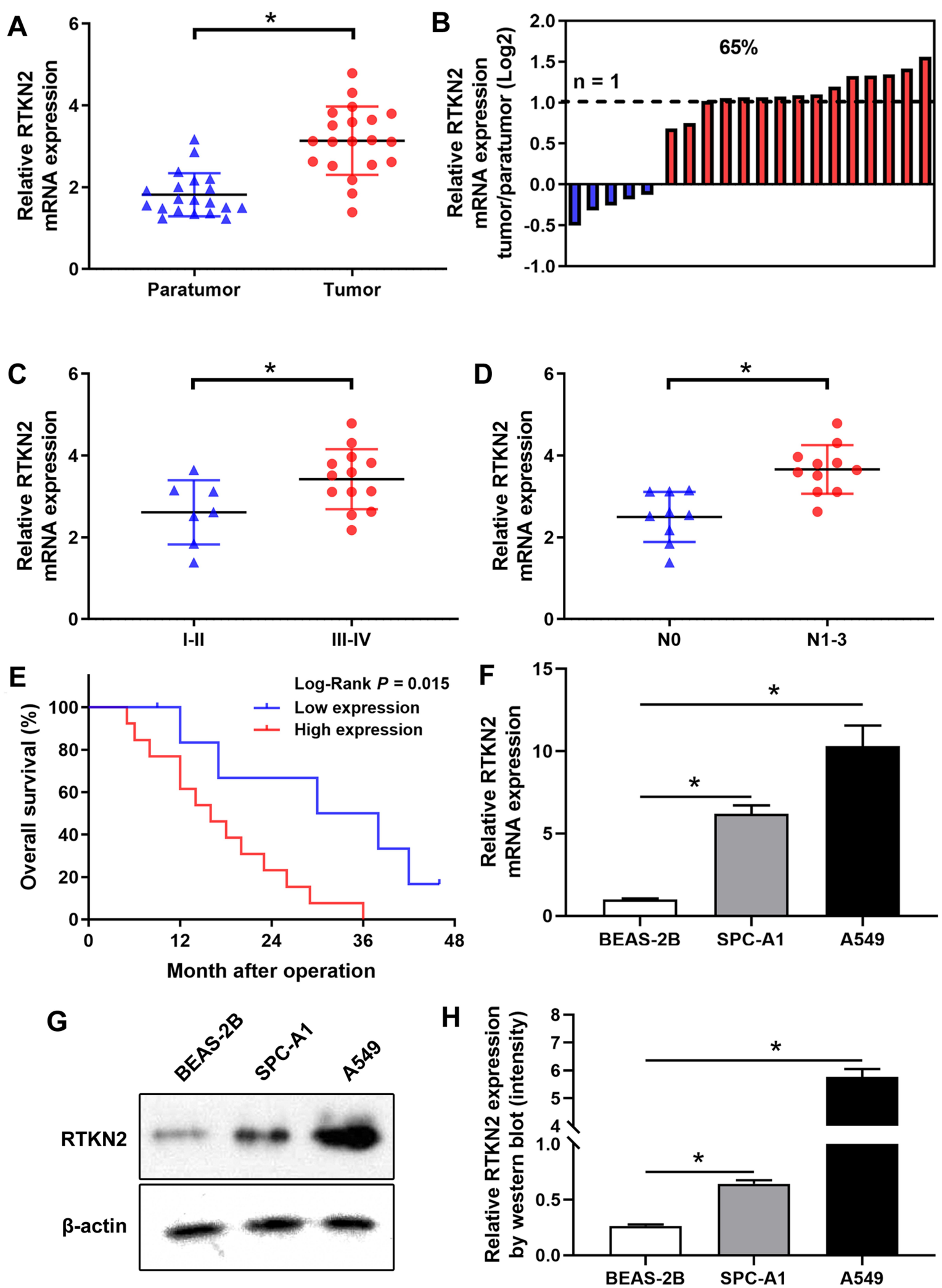

Figure I RTKN2 is up-regulated in NSCLC. (A) The RTKN2 expression level was assessed by qRT-PCR in NSCLC patient tumor and paratumor tissues ( $\mathrm{n}=20$ ). (B) The relative RTKN2 expression was assessed in NSCLC tissues. (C) The expression level of RTKN2 was higher in NSCLC tissues with TNM III-IV. (D) The expression level of RTKN2 was higher in lymph-node positive NSCLC tissues. (E) The RTKN2 high expression group had shorter OS time. (F) Relative mRNA and (G, H) protein expression levels of RTKN2 in NSCLC cell lines (SPC-AI and A549) and normal bronchus epithelium cell line (BEAS-2B). ${ }^{*}<0.05$. 
Table I Association of RTKN2 Expression with Clinicopathological Factors of the NSCLC Patients

\begin{tabular}{|c|c|c|c|c|c|}
\hline \multirow{2}{*}{\multicolumn{2}{|c|}{$\begin{array}{l}\text { Clinicopathological } \\
\text { Variables }\end{array}$}} & \multirow{3}{*}{$\begin{array}{l}\text { n } \\
\\
13\end{array}$} & \multicolumn{2}{|c|}{ RTKN2 Expression } & \multirow{3}{*}{$\begin{array}{l}P \\
\text { Value } \\
0.651\end{array}$} \\
\hline & & & \multirow{3}{*}{$\begin{array}{l}\begin{array}{l}\text { Low } \\
(n=7)\end{array} \\
4 \\
3\end{array}$} & \multirow{3}{*}{$\begin{array}{l}\text { High } \\
(n=13)\end{array}$} & \\
\hline Sex & Male & & & & \\
\hline & Female & 7 & & & \\
\hline \multirow[t]{2}{*}{ Age (years) } & $\leq 60$ & 8 & 3 & 5 & 1.000 \\
\hline & $>60$ & 12 & 4 & 8 & \\
\hline \multirow[t]{2}{*}{ Smoking } & Yes & 8 & 2 & 6 & 0.642 \\
\hline & No & 12 & 5 & 7 & \\
\hline Lymphatic & No & 9 & 6 & 3 & $0.017^{*}$ \\
\hline metastasis & $\mathrm{NI}-3$ & 11 & 1 & 10 & \\
\hline \multirow[t]{2}{*}{ TNM stage } & I and II & 7 & 5 & 2 & $0.022 *$ \\
\hline & III and & 13 & 2 & 11 & \\
\hline
\end{tabular}

Notes: ${ }^{\text {a}}$ Fisher's exact test was used for comparison between subgroups. *Significant result $(P<0.05)$.

The RTKN2 level was markedly higher in A549 cells, so we selected A549 to investigate RTKN2 function in NSCLC.

\section{RTKN2 Silencing Inhibits the Proliferation of A549 Cells}

To explore the function of RTKN2 in NSCLC, siRTKN2 was used to silence RTKN2 in human NSCLC A549 cells with higher RTKN2 level. The efficiency of siRNAs for RTKN2 was confirmed through comparison with si-control at the mRNA level (Figure 2A). Among the three siRNAs, siRNA3 generated the most consistent knockdown efficiency and was chosen for subsequent studies. RTKN2 mRNA (Figure 2A) and protein (Figure 2B) levels were significantly decreased after transfection with siRTKN2 in A549 cells. Next, the MMT assay (Figure 2A) was used to detect proliferation of A549 cells. The results showed that the RTKN2 silencing obviously inhibited the proliferation of A549 cells (Figure 2C). A colony assay (Figure 2D) was carried out to determine the colony forming capacity of A549 cells after RTKN2 silencing. The colonies were significantly decreased in the RTKN2- silencing group compared to the control group.

\section{RTKN2 Knockout Induces Cell Apoptosis of Human NSCLC A549 Cells}

Decreased cell proliferation induced by RTKN2 knockdown may be a consequence of increased cell death.
Thus, we examined the effects of RTKN2 on apoptosis of A549 cells. Annexin V/PI double staining was performed. The apoptosis rate was increased in the siRTKN2-treated A549 cells compared with the si-control group (Figure 3A and B). These data showed that RTKN2 may have an anti-apoptotic role in human NSCLC A549 cells.

\section{Knockdown of RTKN2 Expression Inhibits the Migration and Invasion of A549 Cells}

Cell migration and invasion are critical for metastasis. We evaluated the effect of RTKN2 on the migration and invasion capacity of A549 cells. Transwell experiments demonstrated that knockdown of endogenous RTKN2 expression apparently inhibited the migration and invasion of A549 cells (Figure 4A and B).

\section{Knockdown of RTKN2 Inhibits the Expression Levels of Anti-Apoptosis Protein}

As shown in Figure $5 \mathrm{~A}-\mathrm{C}$, the results indicated that RTKN2 silencing inhibited the expression levels of antiapoptosis protein Bcl-2 and increased the expression of pro-apoptosis protein Bax, which was related to cell apoptosis. It is thought that the ratio of the pro-apoptotic protein Bax to the anti-apoptotic protein Bcl-2 plays a crucial role in the control of the intrinsic pathway of apoptosis. ${ }^{15}$ As shown in Figure 5D, we therefore reasoned that the cell death induced by RTKN2 knockdown may be due to changes in this ratio. These results further showed that RTKN2 had anti-apoptosis potential in human NSCLC cells.

\section{Silencing of RTKN2 Decreases the Expression Levels of MMP9 in A549 Cells} Gelatinases, MMP2 and MMP9, etc., play crucial roles in tumor cell metastasis. Therefore, we evaluated the influence of RTKN2 on the expression level of MMP2 and MMP9. Infection with si-RTKN2 significantly decreased the mRNA expression levels of MMP9 (Figure 6A) compared with the si-control group in A549 cells. This inhibitory effect of RTKN2 silencing was further confirmed by Western blot (Figure 6B and C). Similarly, RTKN2 knockdown remarkably inhibited MMP2 mRNA and protein expression level compared with the si-control group in 

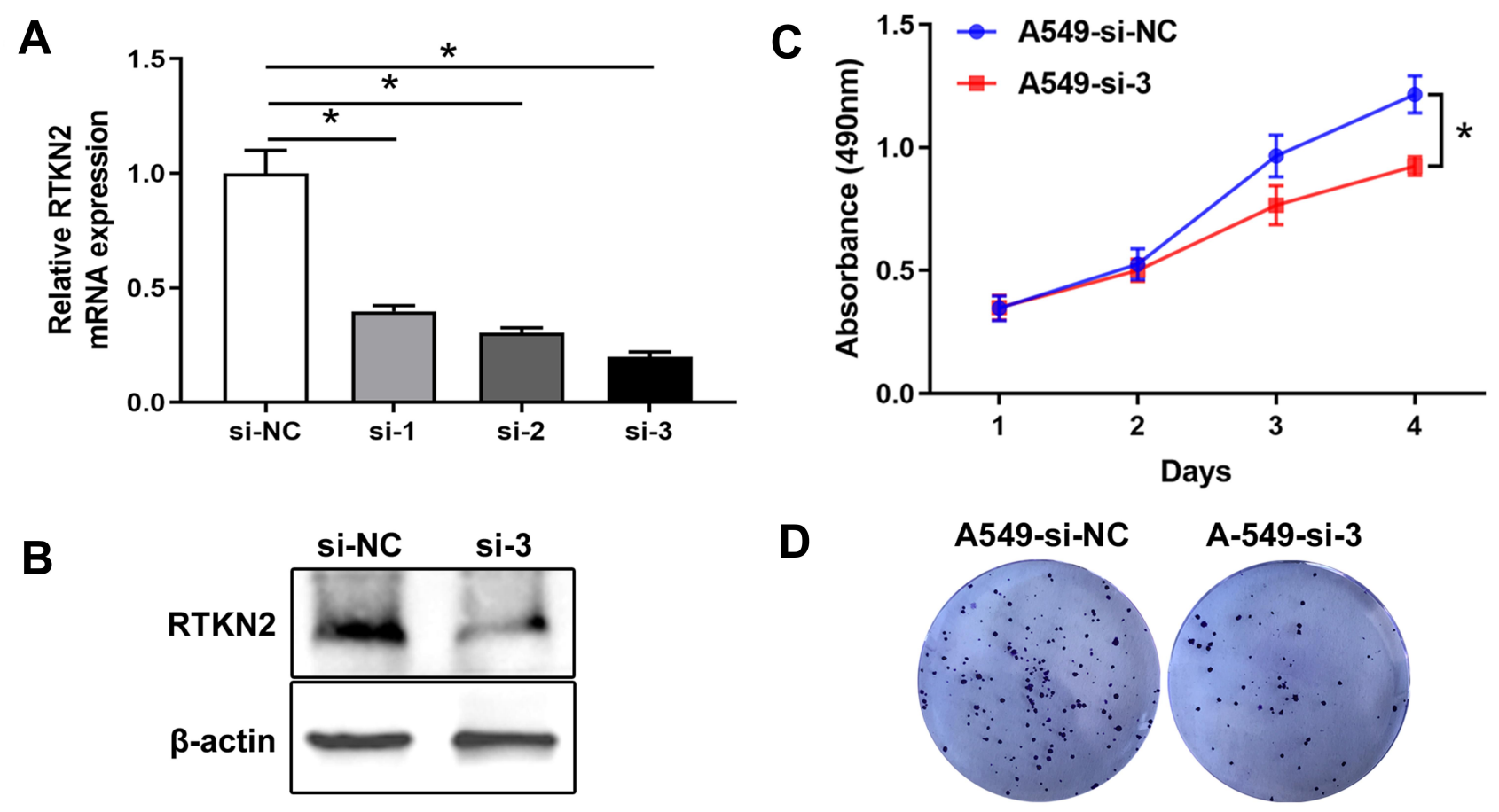

D
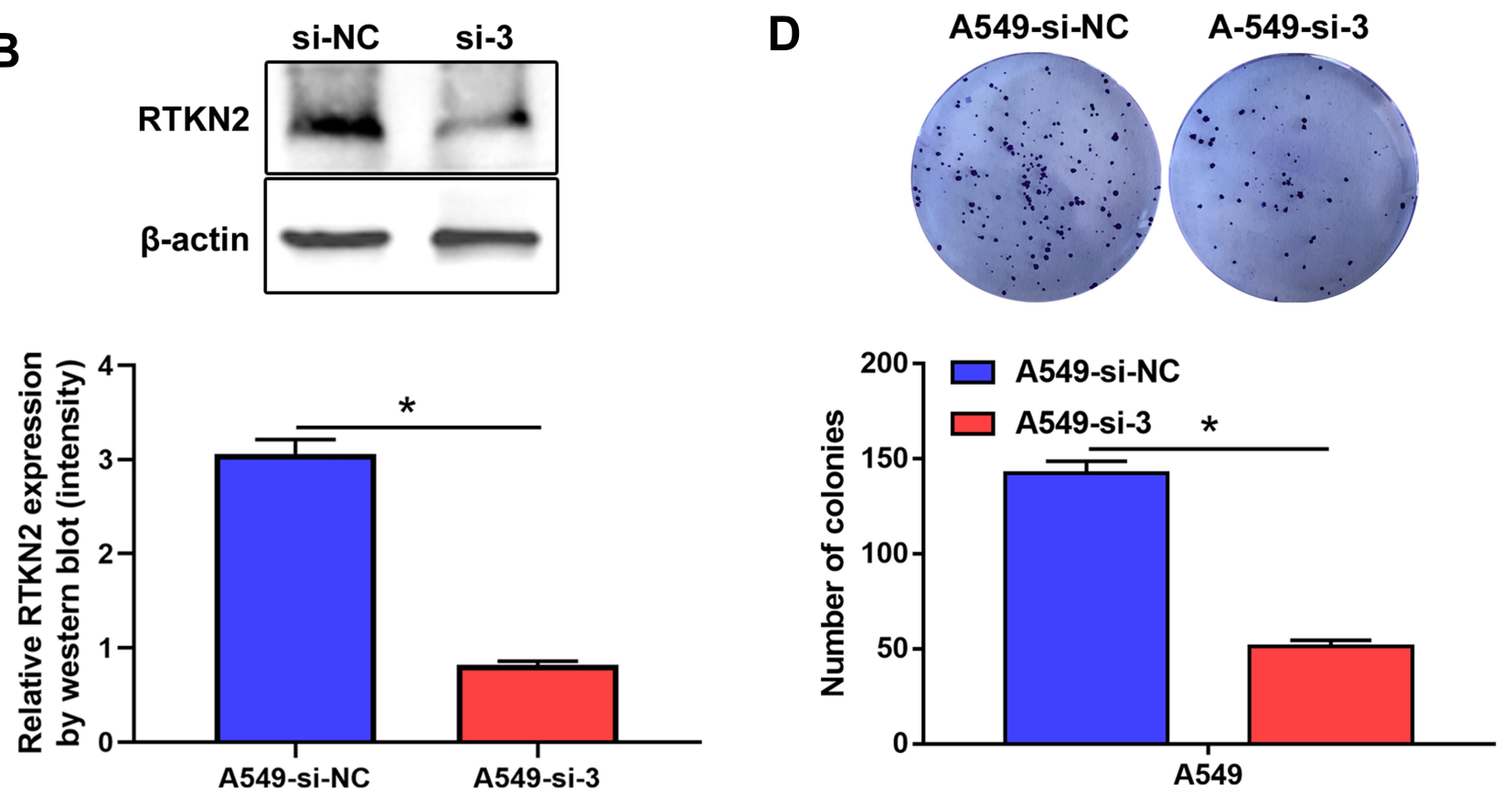

Figure 2 RTKN2 silencing inhibits proliferation of human NSCLC A549 cells. (A-B) RTKN2 was inhibited by different siRNAs in A549 cells. The efficiency of three si RTKN2s was confirmed by qRT-PCR and Western blot assay. (C) MTT cell viability assays were performed $24,48,72$ and $96 \mathrm{~h}$ after the transfection of A549 cells with siRTKN-3. (D) Colony assays were performed. $* P<0.05$.

A549 cells (Figure 6D-F). These data indicate that RTKN2 silencing may inhibit invasion of A549 cells through down-regulating MMP2 and MMP9 expression.

\section{Discussion}

In recent years, despite the development of new multimodal therapeutics, the prognosis for human NSCLC is generally poor. Therefore, it is important to identify additional potential treatments. The Rho GTPases regulate many biological processes, including cell differentiation, survival, transcription, and the cell cycle. ${ }^{8}$ RTKNs are Rho effector proteins, and are expressed in lymphocytes and bone marrow-derived cells, ${ }^{9,16}$ as well as in lung, colon, thymus, and brain. ${ }^{17}$ Two RTKN proteins, RTKN1 and
RTKN2, with the same Rho GTPase binding domain, have homologues in mammals. ${ }^{18}$ RTKN2 may play a critical role in cell proliferation, apoptosis, and metastasis in many malignant tumor cells. The silencing of RTKN2 inhibits proliferation, by reducing the expression levels of MCM10, CDK2, CDC24A, and CDC6 in bladder cancer cells. ${ }^{13}$ Studies have shown that RTKN2 may play a critical role in apoptosis, which is dependent on NF- $x B$ signaling. ${ }^{16,19,20}$ Furthermore, Lin et $\mathrm{al}^{21}$ indicated that miR-181 inhibited the tumorigenesis of ovarian cancer through RTKN2.

In our study, we primarily found that RTKN2 was critical for NSCLC. We analyzed the expression level of RTKN2, and found that RTKN2 was up-regulated in 

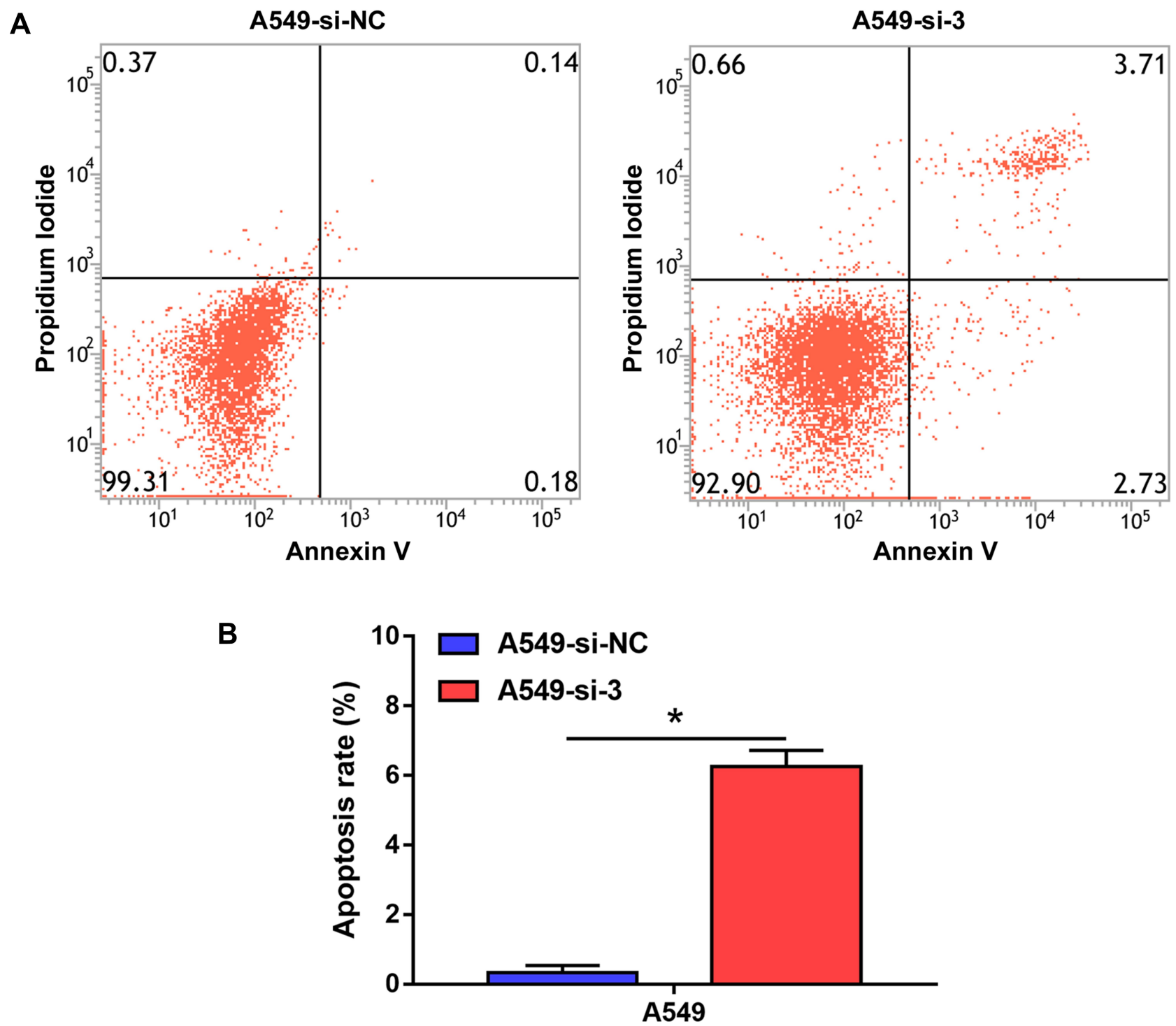

Figure 3 Knockdown of RTKN2 induces cell apoptosis of A549 cells. (A) Apoptosis was analyzed by flow cytometry. (B) Apoptosis was clearly observed in the siRTKN2 group. $* P<0.05$.

NSCLC. TNM stage and lymphatic metastasis were correlated with the RTKN2 mRNA expression level, which demonstrated that RTKN2 was associated with unfavorable prognosis in NSCLC. A functional study by knockdown of RTKN2 expression helped us to illustrate the important role of RTKN2 in NSCLC, where RTKN2 silencing resulted in suppressed proliferation of NSCLC cells and impaired the colony formation ability of NSCLC cells. These results imply that the RTKN2 protein functions as an oncogene and is necessary for human NSCLC cell survival.

Apoptosis is a form of programmed cell death that occurs in multicellular organisms. ${ }^{22}$ The pro-apoptotic protein Bax resides in the cytosol, whereas the anti-apoptotic protein Bcl-2 is localized in the outer mitochondrial membrane, playing a crucial role in the control of the intrinsic pathway of apoptosis. $^{23-26}$ In this study, silencing of RTKN2 increased the level of Bax and the ratio of Bax/ Bcl-2, and decreased the expression level of Bcl-2.

Multiple studies have observed a significant increase in MMP9 in NSCLC, and MMP9 performs important roles in tumor metastasis. ${ }^{27,28}$ In our study, we found that RTKN2 knockout significantly inhibited the MMP9 and MMP2 expression levels of A549 cells. The results demonstrated that RTKN2 promoted metastasis of NSCLC cells through up-regulated MMP9 and MMP2 expression levels.

In summary, we demonstrated that RTKN2 knockout obviously inhibited the proliferation of human NSCLC A549 

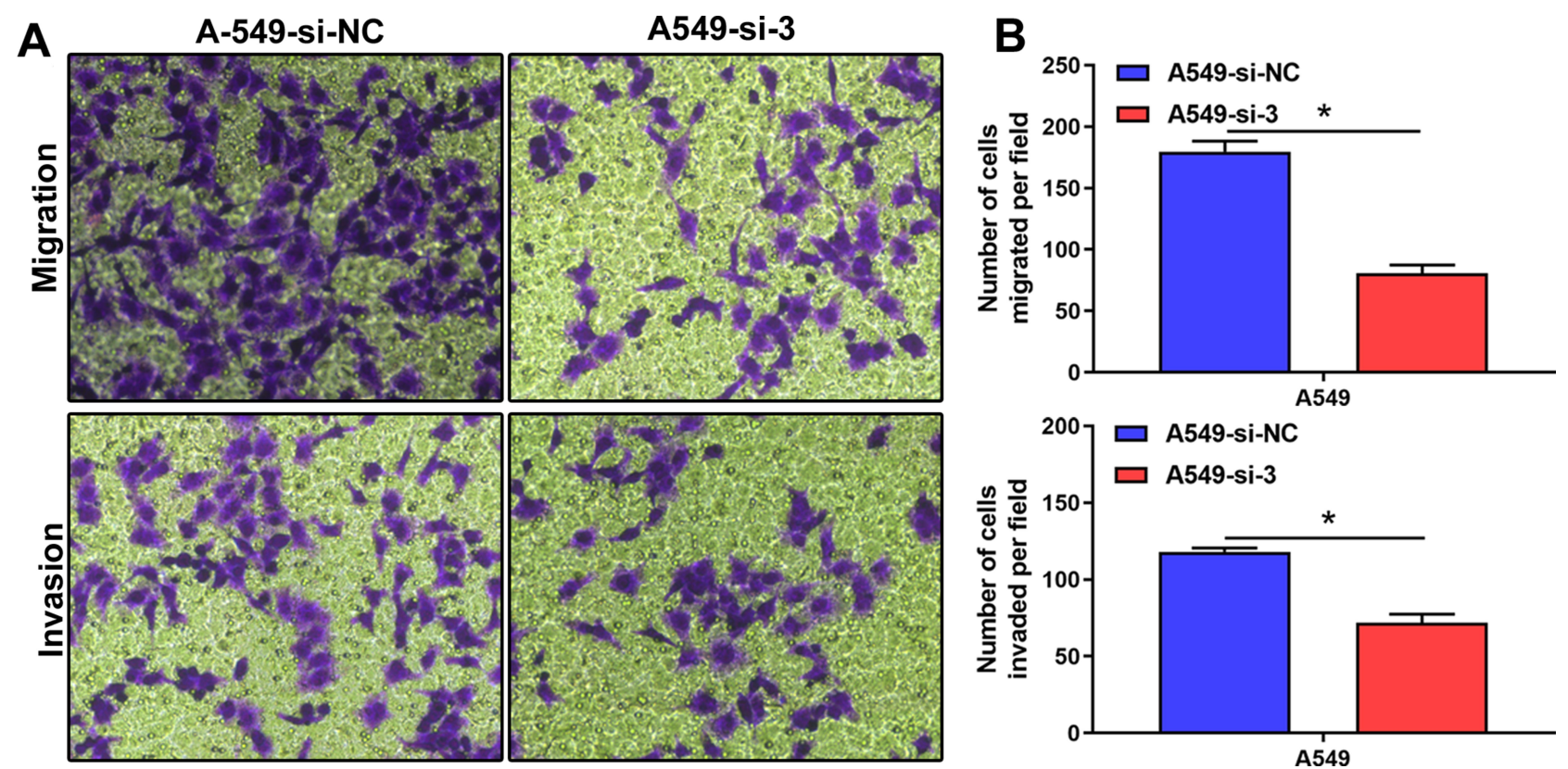

Figure 4 Depletion of RTKN2 suppresses migration and invasion of A549 cells. (A) Transwell assays were performed for migration and invasive ability of A549 cells. (B) The numbers of migrated and invasive cells were counted. $* P<0.05$.

A
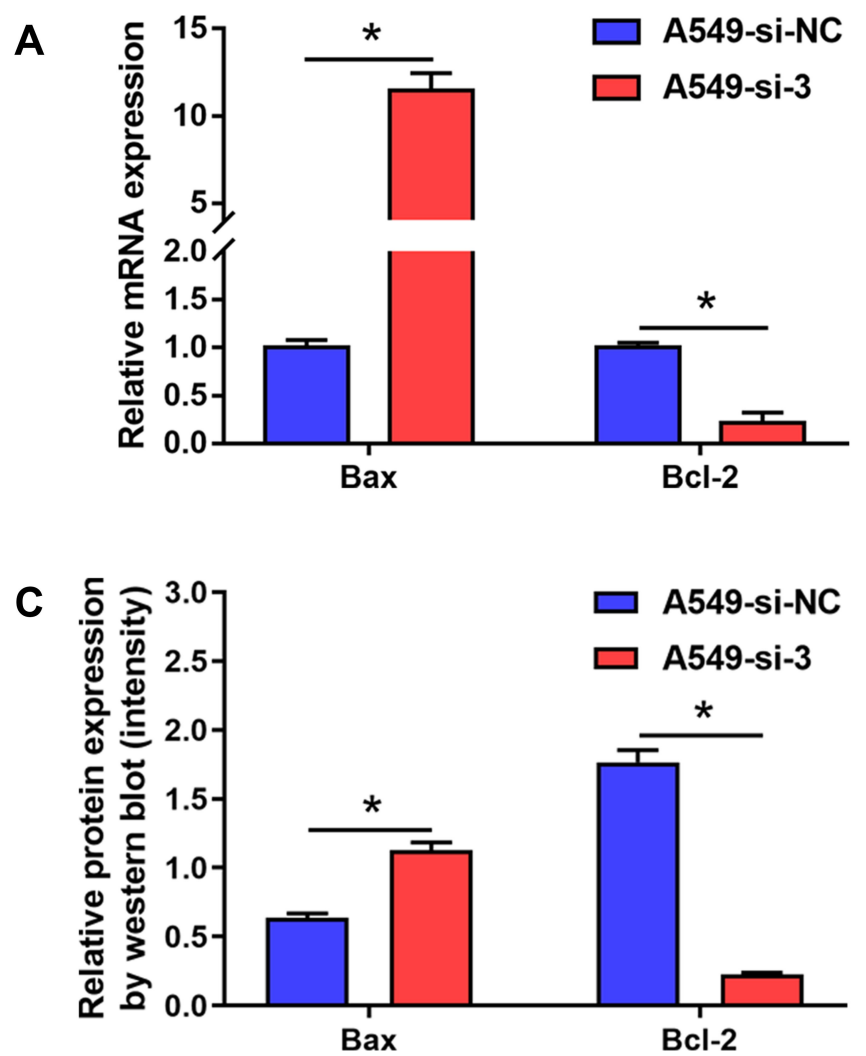

B
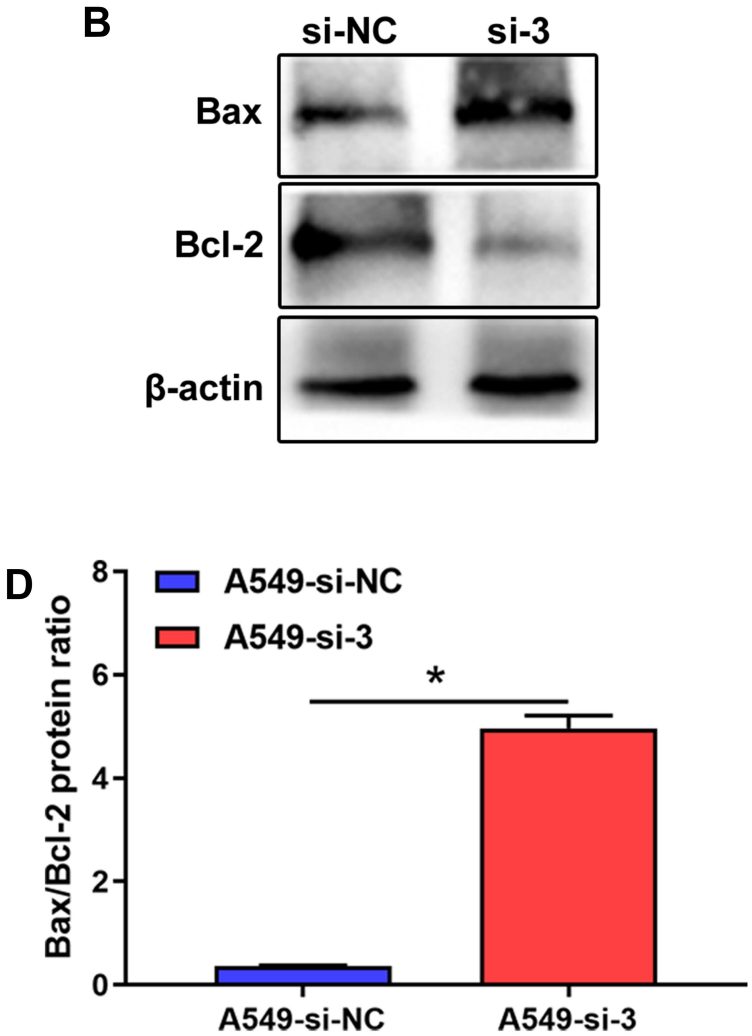

Figure 5 Expression level of apoptosis-related proteins. (A) qRT-PCR (B) and Western blot showed a significant increase in the expression level of Bax, and decreased expression level of Bcl-2 in A549 cells transfected with siRTKN2. (C) The Western blot was quantitatively analyzed by Imagej. (D) Ratio of Bax/Bcl-2 level. $* P<0.05$. 

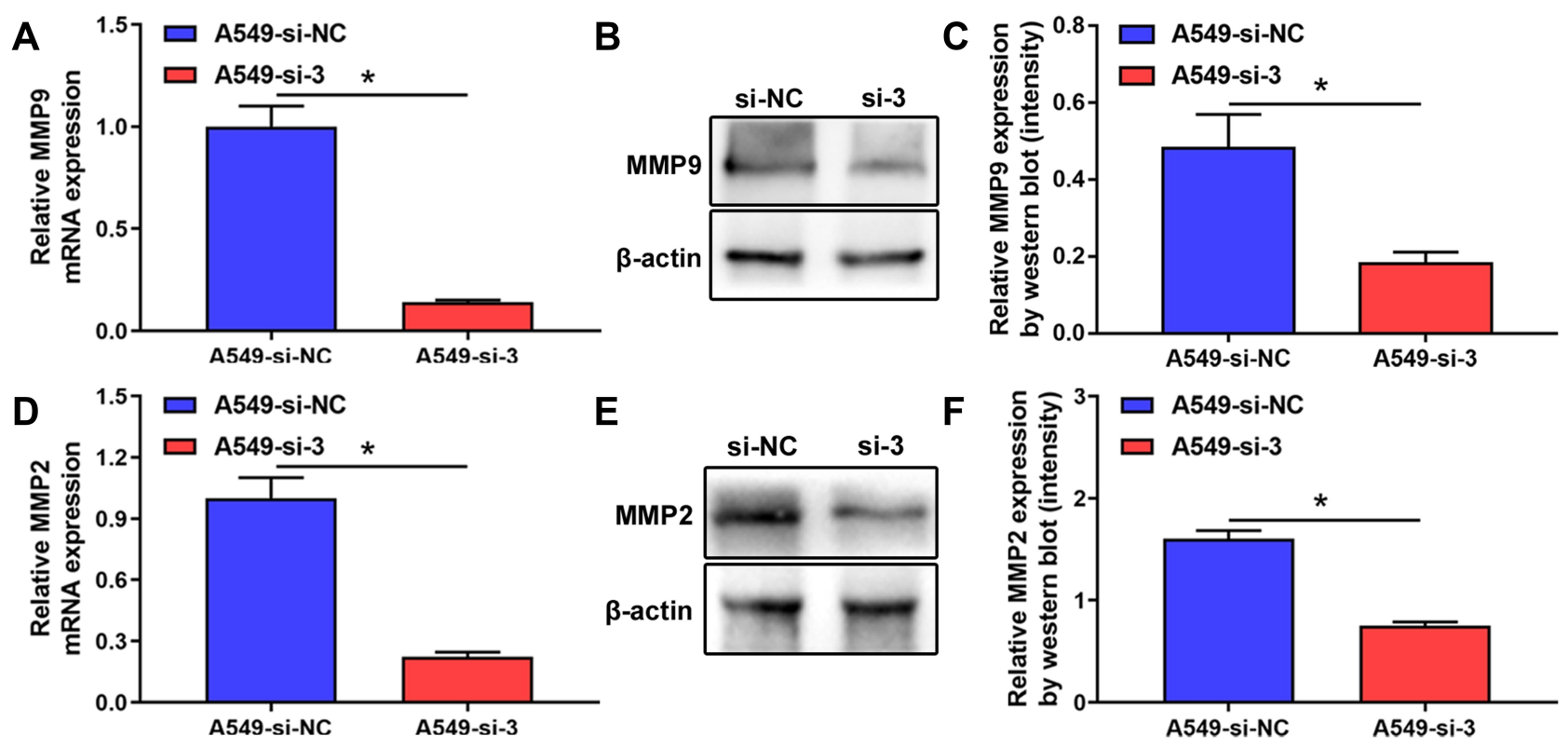

E
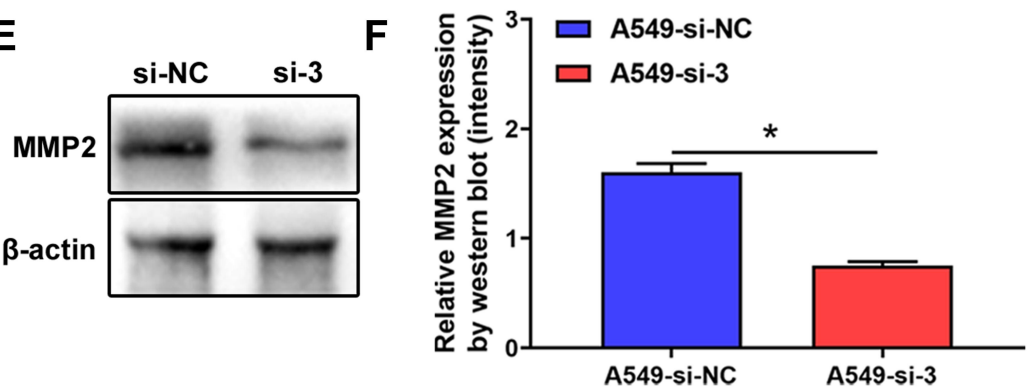

Figure 6 Knockdown of RTKN2 reduces the MMP9 level of A549 cells. (A) qRT-PCR (B) and Western blot assay showed a significant decrease in the MMP9 level in A549 cells transfected with RTKN2-siRNA. (C) The Western blot was quantitatively analyzed by densitometry. (D) qRT-PCR (E) and Western blot assay showed a significant decrease in the MMP2 level in A549 cells transfected with siRTKN2. (F) The Western blot was quantitatively analyzed by densitometry. $* P<0.05$.

cells through inducing apoptosis of NSCLC cells, and significantly suppressed the migration and invasion of NSCLC cells. These data suggest that RTKN2 may not only be a prognostic biomarker candidate but also provide a potential therapeutic target for NSCLC diagnosis and treatment.

\section{Abbreviations}

NSCLC, non-small-cell lung cancer; RTKN2, Rhotekin 2; FBS, fetal bovine serum; MMP9, matrix metalloproteinase 9; PI, propidium iodide.

\section{Funding}

The present study was supported by the Medical Scientific Research Foundation of Zhuhai, China (Grant ID, 2016J025).

\section{Disclosure}

The authors report no conflicts of interest in this work.

\section{References}

1. Siegel RL, Miller KD, Jemal A. Cancer Statistics, 2017. CA Cancer J Clin. 2017;67(1):7-30. doi:10.3322/caac.21387

2. Remon J, Besse B. Immune checkpoint inhibitors in first-line therapy of advanced non-small cell lung cancer. Curr Opin Oncol. 2017;29 (2):97-104. doi:10.1097/CCO.0000000000000351

3. Weichert W, Warth A. Early lung cancer with lepidic pattern: adenocarcinoma in situ, minimally invasive adenocarcinoma, and lepidic predominant adenocarcinoma. Curr Opin Pulm Med. 2014;20 (4):309-316. doi:10.1097/MCP.0000000000000065
4. Chen Z, Fillmore CM, Hammerman PS, et al. Non-small-cell lung cancers: a heterogeneous set of diseases. Nat Rev Cancer. 2014;14 (8):535-546. doi:10.1038/nrc3775

5. Reck M, Heigener DF, Mok T, et al. Management of non-small-cell lung cancer: recent developments. Lancet. 2013;382(9893):709-719. doi:10.1016/s0140-6736(13)61502-0

6. Hirsch FR, Scagliotti GV, Mulshine JL, et al. Lung cancer: current therapies and new targeted treatments. Lancet. 2017;389:299-311. doi:10.1016/s0140-6736(16)30958-8

7. Herbst RS, Morgensztern D, Boshoff C. The biology and management of non-small cell lung cancer. Nature. 2018;553:446-454. doi:10.1038/nature25183

8. Bishop AL, Hall A. Rho GTPases and their effector proteins. Biochem J. 2000;348(Pt 2):241-255. doi:10.1042/bj3480241

9. Reid T, Furuyashiki T, Ishizaki T, et al. Rhotekin, a new putative target for Rho bearing homology to a serine/threonine kinase, PKN, and rhophilin in the rho-binding domain. $J$ Biol Chem. 1996;271 (23):13556-13560. doi:10.1074/jbc.271.23.13556

10. Pang X, Li R, Shi D, et al. Knockdown of Rhotekin 2 expression suppresses proliferation and induces apoptosis in colon cancer cells. Oncol Lett. 2017;14:8028-8034. doi:10.3892/ol.2017.7182

11. Wang X, Zhang L, Wang W, et al. Rhotekin 2 silencing inhibits proliferation and induces apoptosis in human osteosarcoma cells. Biosci Rep. 2018:38. doi:10.1042/BSR20181384

12. Wei W, Chen H, Liu S. Knockdown of Rhotekin 2 expression suppresses proliferation and invasion and induces apoptosis in hepatocellular carcinoma cells. Mol Med Rep. 2016;13(6):4865-4871. doi:10.3892/mmr.2016.5113

13. Liao YX, Zeng JM, Zhou JJ, et al. Silencing of RTKN2 by siRNA suppresses proliferation, and induces G1 arrest and apoptosis in human bladder cancer cells. Mol Med Rep. 2016;13(6):4872-4878. doi: $10.3892 / \mathrm{mmr} .2016 .5127$

14. Zhang L, Chen J, Yong J, et al. An essential role of RNF187 in Notch1 mediated metastasis of hepatocellular carcinoma. $J$ Exp Clin Cancer Res. 2019;38(1):384. doi:10.1186/s13046-019-1382-x

15. Zhang L, Li Y, Qiao L, et al. Protective effects of hepatic stellate cells against cisplatin-induced apoptosis in human hepatoma G2 cells. Int $J$ Oncol. 2015;47(2):632-640. doi:10.3892/ijo.2015.3024 
16. Collier FM, Loving A, Baker AJ, et al. RTKN2 Induces NF-KappaB Dependent Resistance to Intrinsic Apoptosis in HEK Cells and Regulates BCL-2 Genes in Human CD4 + Lymphocytes. J Cell Death. 2009;2:9-23. doi:10.4137/jcd.s2891

17. Yin T-Y, Hsiao Y-W, Peng W-H, et al. Abstract 4435: overexpression of Rhotekin confers gastric cancer cells resistance to interferon- $\alpha$ mediated growth inhibition. Cancer Res. 2012;72:4435. doi:10.1158/ 1538-7445.am2012-4435

18. Collier FM, Gregorio-King CC, Gough TJ, et al. Identification and characterization of a lymphocytic Rho-GTPase effector: rhotekin-2. Biochem Biophys Res Commun. 2004;324(4):1360-1369. doi:10. 1016/j.bbrc.2004.09.205

19. Liu CA, Wang MJ, Chi CW, et al. Rho/Rhotekin-mediated NFkappaB activation confers resistance to apoptosis. Oncogene. 2004;23(54):8731-8742. doi:10.1038/sj.onc.1208106

20. Myouzen K, Kochi Y, Okada Y, et al. Functional variants in NFKBIE and RTKN2 involved in activation of the NF-kappaB pathway are associated with rheumatoid arthritis in Japanese. PLoS Genet. 2012;8 (9):e1002949. doi:10.1371/journal.pgen.1002949

21. Lin Z, Li D, Cheng W, et al. MicroRNA-181 Functions as an Antioncogene and Mediates NF-kappaB Pathway by Targeting RTKN2 in Ovarian Cancers. Reprod Sci. 2019;26(8):1071-1081. doi:10.1177/1933719118805865
22. Korsmeyer SJ. Regulators of cell death. Trends Genetics. 1995;11 (3):101-105. doi:10.1016/s0168-9525(00)89010-1

23. Martinou J-C, Youle RJ. Mitochondria in Apoptosis: bcl-2 Family Members and Mitochondrial Dynamics. Dev Cell. 2011;21(1):92101. doi:10.1016/j.devcel.2011.06.017

24. Czabotar PE, Lessene G, Strasser A, et al. Control of apoptosis by the BCL-2 protein family: implications for physiology and therapy. Nat Rev Mol Cell Biol. 2014;15:49-63. doi:10.1038/nrm3722

25. Chipuk JE, Moldoveanu T, Llambi F, et al. The BCL-2 Family Reunion. Mol Cell. 2010;37(3):299-310. doi:10.1016/j.molcel.2010. 01.025

26. Zhang L, Yu J, Park BH, et al. Role of BAX in the apoptotic response to anticancer agents. Science. 2000;290(5493):989. doi:10.1126/ science.290.5493.989

27. Zheng P, Wang W, Ji M, et al. TMEM119 promotes gastric cancer cell migration and invasion through STAT3 signaling pathway. Onco Targets Ther. 2018;11:5835-5844. doi:10.2147/ott.s164045

28. Chien YC, Liu LC, Ye HY, et al. EZH2 promotes migration and invasion of triple-negative breast cancer cells via regulating TIMP2MMP-2/-9 pathway. Am J Cancer Res. 2018;8:422-434.

\section{Publish your work in this journal}

OncoTargets and Therapy is an international, peer-reviewed, open access journal focusing on the pathological basis of all cancers, potential targets for therapy and treatment protocols employed to improve the management of cancer patients. The journal also focuses on the impact of management programs and new therapeutic agents and protocols on patient perspectives such as quality of life, adherence and satisfaction. The manuscript management system is completely online and includes a very quick and fair peer-review system, which is all easy to use. Visit http://www.dovepress.com/ testimonials.php to read real quotes from published authors. 